https://www.journal-imab-bg.org

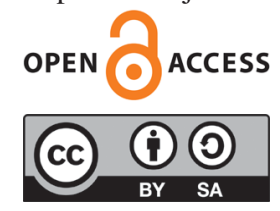

Review article

\title{
INFLUENZA INFECTION AS TRIGGER FOR ACUTE MYOCARDIAL INFARCTION AND STROKE
}

\author{
Darina Miteva \\ Clinic of Pneumology and Phthysiatry, UMHAT St. Marina, Department of \\ Pulmonology and Allergology, Faculty of Medicine, Medical University, Varna, \\ Bulgaria.
}

\begin{abstract}
:
Influenza affects millions of patients every year. The manifestations vary from mild respiratory symptoms to severe pulmonary and extra-pulmonary complications and even death. Recent scientific evidence has been accumulating that influenza infection can trigger acute myocardial infarction and stroke. Both diseases are the leading causes of death worldwide, and the recognition of the risk factors is crucial for adequate prevention. This article reviews the pathogenetic links between influenza and the development of myocardial infarction and stroke. It presents the incidence, timing and outcome of these acute cardiovascular events after influenza infection and the role of vaccination for their prevention. The aim of the review is to focus the attention of medical practitioners on the association between influenza and cardiovascular events in order to improve the prevention and management of highrisk patients.
\end{abstract}

Keywords: respiratory infections, pneumonia, influenza, acute myocardial infarction, stroke

\section{BACKGROUND}

Influenza is one of the most common infectious diseases affecting millions of patients every year. It usually presents with mild respiratory symptoms, but can also lead to severe pulmonary and extra-pulmonary complications and even death. According to WHO data, the annual epidemics lead to about 3 to 5 million cases of severe illness, and about 290000 to 650000 respiratory deaths worldwide [1]. Influenza complications include both respiratory and extra-respiratory manifestations. Respiratory complications include otitis media, sinusitis, bronchitis, in young children bronchiolitis, exacerbation of chronic lung diseases as bronchial asthma and COPD, primary influenza pneumonia and secondary bacterial pneumonia. Serious extra-pulmonary complications include cardiovascular manifestations as myocarditis and pericarditis, neurological complications such as meningitis and encephalitis, renal failure and others. On the other hand, ischaemic heart disease (IHD) and stroke are the leading causes of mortality worldwide. In 2016 they led to combined 15.2 million deaths [2]. In addition to increased mortality, acute cardio- vascular events (CVE) can also lead to increased disability. The classic risk factors include hypertension, dyslipidemia, diabetes mellitus, smoking. Recently scientific evidence is increasing that infections and above all respiratory infections can trigger acute myocardial infarction (AMI) and stroke. Some scientific reports consider pneumonia as such triggering factor. But there are also a number of studies proving that the risk of AMI and stroke is increased not only in bacterial pneumonia but also inviral infections, especially influenza, which have not led to the development of pneumonia. The recognition of the additional and potentially preventable risk factors can decrease the incidence and improve the prognosis for these acute cardiovascular events.

\section{Pathogenetic mechanisms of association between influenza and CVE}

Atherosclerosis involves middle and large arteries and is characterized by the formation of atherosclerotic plaques consisting of a lipid-rich core and fibrous cap. For years, plaques can remain stable. Various factors can make plaque vulnerable and unstable. They lead to edema, increased intraplaque pressure and formation of necrosis, hemorrhage, erosion and rupture of the plaque. Activated platelets are deposited, and acute thrombus is formed, which is crucial for the acute complications of atherosclerosis such as acute coronary syndrome and ischemic stroke. Respiratory infections can lead to plaque instability by various mechanisms. In animal models, the infection with influenza A virus induced subendothelial infiltrate composed of smooth muscle cells, macrophages, and CD3 T lymphocytes. Platelet and fibrin-rich thrombus leading to subtotal stenosis were also observed [3]. Infection can lead to systemic inflammation and increase of inflammatory cells and pro-inflammatory biomarkers in the circulation such as C-reactive protein, procalcitonin, IL-6, TNF- $\alpha$, and others. Atherosclerotic plaques are infiltrated by inflammatory cells which produce various cytokines, proteases, coagulation factors, oxygen radicals and vasoactive substances which stimulate plaque instability and acute thrombus formation [4]. The prothrombotic state, as part of the systemic inflammatory response syndrome, also plays a role for in the formation of acute thrombus. On the one hand, direct 
activation of platelets by products released from pathogenic microorganisms may occur. On the other hand, changes in the activity and concentrations of coagulation factors as antithrombin III, activated protein $\mathrm{C}$ and plasminogen activator-1 inhibitor may also contribute to the development of a procoagulant state. The increased sympathetic activity leads to vasoconstriction, tachycardia and cardiac arrhythmias. The hypotension and hypoxemia also aggravate the oxygen demand/supply mismatch and induce myocardial and cerebral ischaemia. The risk of AMI and stroke is particularly increased in pneumonia. Aliberti and Ramirez divide the pathologic mechanisms of cardiovascular events in pneumonia into plaque-related and plaqueunrelated mechanisms [5]. Both AMI and stroke belong to the plaque-related complications as they share similar pathophysiological mechanisms related to plaque instability and oxygen demand/supply imbalance. The risk of developing acute coronary or cerebral ischemia is increased not only in pneumonia but also in mild and moderate respiratory infections as bronchitis, pharyngitis, rhinitis or sinusitis. An increase of proinflammatory markers and development of the procoagulant state is also observed in uncomplicated viral infections [6,7]. The pathogenetic links between respiratory infections and acute coronary syndrome are presented in detail in the excellent papers of Corrales-Medina and co-authors [8,9] and Bazazand coauthors [10]. The different aspects of the association between infection and stroke are discussed in the review of Miller and co-workers [11]. As mentioned above, similar pathogenic mechanisms play a role as triggers for both diseases.

\section{AMI and stroke after influenza: incidence, timing and outcome}

In 1932 Collins first described the increase in mortality due to influenza and other non-respiratory causes during winter suggesting a causal relationship between them [12]. After that, a number of other studies have established an increase in the number of deaths from IHD and cerebrovascular disease in the winter, which correlates with the influenza activity $[13,14]$. This association was especially strong for myocardial infarction mortality which increases between $5.8 \%$ and $13.1 \%$ with each interquartile range increase in influenza incidence during the previous 14 days [15]. In more than 34000 autopsies between 1993 and 2000, Madjid and co-authors found a peak of AMI and chronic IHDmortality during acute respiratory disease activity, including influenza[16]. The data about the seasonal link between influenza and stroke are more controversial. Foster and co-workers, for instance, found seasonal relationship only between AMI and influenza, but not between ischaemic strokes and influenza activity [17]. The highest risk of AMI and stroke is observed close after the infection, within the first 3 days to 1-2 weeks, but it remains significantly elevated within 3 months $[18,19,20,21]$. Other authors found shorter periods of association between influenza infection and CVE -the elevated risk of myocardial infarction for about 1 week and of stroke about 28 days [22]. Smeeth and co-workers conductåd a study including more than 20,000 patients with a first AMI and more than 19,000 patients with the first stroke and found elevated risk for occurrence of both events after a respiratory tract infection (pneumonia, acute bronchitis, "chest infections," and influenza). Within the first 3 days after infection, the risk for AMI was almost 5-fold higher and for stroke about 3 -fold higher then it fell gradually but still remained elevated within the next three months. [18]. Clayton et al. found a 2-fold increased risk of AMI and stroke within the first 7 days of the onset of respiratory infection, as the risk for MI was elevated independently of the initial underlying cardiovascular risk [19]. During infection, the increase in troponin and the occurrence of some ECGabnormalities may be due to non-ischemic causes, e.g. myocarditis. To eliminate this possibility, Ruane et al. investigated only patients with angiographically confirmed AMI. Symptoms of respiratory infection had $17 \%$ of the patients within 7 days and $21 \%$ of the patients within 35 days bevor the onset of MI. The relative risk for MI during the first week was significantly increased even in patients with milder, upper respiratory tract infection, with RR 13.5 (95\% CI 10.2-17.7) [23].

Many studies examining the relationship between respiratory infection and AMI and stroke have no etiological confirmation of the infection. Therefore, studies that report data on a specific etiologic agent as a trigger factor are of particular interest. Using data from the Scottish Morbidity RecordWarren-Gashand co-workers conducted a self-controlled case series study and evaluated the risk for first AMI or stroke after laboratory-confirmed respiratory infections. The most common respiratory pathogens from the bacterial and viral origin are included in the analysis. The influenza virus increased the risk of myocardial infarction to a greater degree than S. pneumoniae: adjusted IRs for the first 1-3 days after infection were 9.80 (95\% CI 2.37-40.5) and 5.98 (95\% CI 2.47-14.4), respectively. Inversely, the risk of stroke was higher for $\mathrm{S}$. pneumoniae than influenza virus for day 1-3: adjusted IRs 12.3 (95\% CI 5.48-27.7) and 7.82 (95\% CI 1.07-56.9), respectively. Other respiratory viruses (human metapneumovirus, parainfluenza, respiratory syncytial virus, rhinovirus) also increased the risk of AMI and stroke, but to a lesser degree. Their combined effect as triggers of AMI and stroke was less than that of influenza alone [22]. Similar results were found in another self-controlled caseseries study from Canada evaluating the connection between confirmed influenza infection and hospitalization for AMI. Patients with influenza had an approximately 6-fold increased risk of admission for AMI within the first 7 days from detection of infection, with no increased risk after this interval. Other viruses, such as RSV, also increased this risk, although to a lesser degree. Higher incidence of AMI was observed in older patientse" 65 years of age[24]. Regarding the age groups at elevated risk for developing CVE after a respiratory infection, the results are controversial. Blackburn found significantly increased hospitalizations for AMI and ischaemic stroke in patients aged $\geq 75$ years after respiratory viral infection. At the age of 65-74 years, most respiratory viruses increased hospitalizations for MI, but not an ischemic stroke. At the age of 45-64, respiratory infections were not 
associated with increased hospitalization for both diseases [25]. Contrariwise Warren-Gash and co-workers found that younger patients $<65$ years were prone tohigherAMI and stroke risk compared to those aged $\geq 65$ years, the authors speculated that this might be related to the lower vaccination rate among them [22]. Other studies also demonstrated that recent influenza like illness has a greater impact as a risk factor for stroke in younger patients under 45 years compared to older patients. This may be related to the smaller impact of the conventional risk factors on the risk of stroke in younger patients [26]. Stroke is very rare in children, but an international case-control study found a 6-fold increased risk of ischemic stroke in children suffering from an infection, mainly respiratory, in the week prior to the stroke. The stroke risk was higher in unvaccinated or partly vaccinated children compared to those receiving all or most routine vaccinations [27].

Another interesting question is whether patients with AMI and stroke after influenza have a worse prognosis than those without an infection. Vejpongsa and co-workers conducted a study over more than 1,800,000 hospitalized patients with AMI, of whom $1.3 \%$ had influenza or other viral respiratory infection. Patients with AMI after influenza had not only higher in-hospital mortality but also higher rate of other adverse outcomes (defined as a shock, respiratory failure, acute kidney injury, and prolonged hospital stay) compared to patients without infection or with a noninfluenza viral respiratory infection. The AMI patients with influenza underwent invasive coronary angiography more rarely, and this could be one of the potential causes for the worse outcome [28]. Among hospitalized patients with AMI Putot and co-workers found $10 \%$ incidence of concurrent acute infection, $67 \%$ of whom were respiratory infections (acute bronchitis and pneumonia). Only a small number of patients had microbiological identification of the causative pathogen. The clinical presentation of post-infectious MI was more severe, and the in-hospital mortality rate was higher $(11$ vs $6 \%, \mathrm{p}<0.01)$. The post-infectious MI was more often type 2 (due to myocardial oxygen demand/supply mismatch), but type 1 post-infectious MI (due to acute plaque disruption) had a worse prognosis. Compared to another site of infection, respiratory tract infections led to about threefold increased risk of hospital mortality [29].

\section{against CVE \\ Influenza vaccination as a preventive strategy}

Multiple studies have reported the benefit of influenza vaccination on mortality and non-fatal CVE in patients with underlying cardiovascular disease. The FLUVACS study revealed a favourable effect of vaccination on the cardiovascular mortality in patients with IHD as the positive effect was observed mainly in patients with AMI [30]. A study from Taiwan found that in elderly patients, with previous myocardial infarction vaccination decreased significantly, the incidence of recurrent infarction or cardiovascular death during the 1-year follow-up period [31]. Meta-analysis of randomised controlled trials found that vaccination was associated with a lower risk of composite major adverse CVE, especially in high-risk patients with recent acute coronary syndrome within 1 year [32]. These results emphasise that among patients with IHD, the greatest benefit from vaccination have these with more active coronary disease. A review of randomised controlled trials revealed positive effects of influenza vaccination on the cardiovascular mortality (RR $0.45,95 \%$ CI 0.26 to 0.76 , $\mathrm{P}=0.003$ ) and on the incidence of combined acute CVE in patients with underlying cardiovascular disease (a role for secondary prevention). In the general population, there were no significant differences between vaccinated and unvaccinated groups, and influenza vaccination failed to show a positive effect in primary prevention. Regarding myocardial infarction, no significant difference was found between the compared groups [33]. Different results come from case control studies. MacIntyre and co-workers found vaccine effectiveness of $45 \%$ as a protective measure against AMI [34] and in a meta-analysis of case-control studies, the vaccine effectiveness was estimated of $29 \%$ [35]. This efficacy is comparable with the standard coronary artery disease prevention strategy: smoking cessation (32-43\%), statins (19$30 \%)$ and antihypertensive therapy (17-25\%) [36].

Research findings for the effectiveness of influenza vaccination as a preventive measure against stroke are also controversial. According to some studies, vaccination reduces the risk of stroke $[37,38]$, while others fail to find such a benefit [39]. Positive effect was established even with one vaccination, but the regular annual revaccination decreased the risk of ischemic stroke admissions at a higher degree [38]. Some studies found positive effects only in ischemic stroke[37], while others found that vaccination decreased the risk for all subtypes of strokes [40]. A population-based cohort study which included patients $\geq 65$ years old who were treated in intensive care unit in Denmark found that influenza vaccination reduced the risk of stroke (adjusted HR, 0.84; 95\% CI 0.78-0.92), during the 1-year follow-up period, but the reduction of the risk of myocardial infarction was non-significant. The vaccinated ICU survivors also had a lower all-cause mortality compared with the unvaccinated [41]This study highlights again the positive role of vaccination for the most vulnerable and fragile patients.

The mechanism of the protective effect of influenza vaccination on the CVE has not been fully clarified. The benefit of vaccination is not limited to decreasing the incidence of influenza infection and its complications. In mice models, Bermudez-Fajardo found that vaccinated animals developed more stable plaque phenotype with lower lipid content. There were also some differences in the cytokines production as the vaccinated mice showed lower levels of interferon gamma, IL-2 and TNF $\alpha$ but higher levels of IL-4. These results show that vaccination may exert a protective effect against cardiovascular disease by contributing to the development of smaller and more stable plaques and by alterations in the immune response to the infection [42]. Veljkovic and co-workers identified the bradykinin 2 receptor (BKB2R) as the main protein involved in the intimate molecular processes through which the vaccine exerts its cardioprotective effect. Postvaccination antibodies may activate the BKB2R, and this may in- 
crease the levels of nitric oxide, leading to vasodilation and possible angiogenesis and through to improved myocardial oxygen use. [43] Future studies are needed to shed more light on these complicated pathogenetic mechanisms.

Despite the benefits of influenza vaccination, in many countries, including Bulgaria, it is still underused [44]. Its additional benefits in preventing cardiovascular events are another argument in favor of its wider application in indicated individuals.

\section{CONCLUSION}

Influenza infection increases the incidence of CVE, especially in the first days and weeks after infection and worsens their clinical course making the prognosis more severe. Being familiar with the association between influenza and AMI and stroke, clinicians will be able to promote vaccination more actively and monitor high-risk groups more closely. In this way, they can improve the prevention and management of the influenza infection and reduce its complications and mortality.

\section{REFERENCES:}

1. Influenza (Seasonal). WHO. 6 November 2018. [Internet]

2. The top 10 causes of death. WHO. 24 May 2018. [Internet]

3. Naghavi M, Wyde P, Litovsky S, Madjid M, Akhtar A, Naguib S, et al. Influenza infection exerts prominent inflammatory and thrombotic effects on the atherosclerotic plaques of apolipoprotein E-deficient mice. Circulation. 2003; 107(5):762-68.

4. Hansson GK, Robertson AK, Soderberg-Naucler C. Inflammation and atherosclerosis. Annu Rev Pathol. 2006; 1: 297-329.

5. Aliberti S, Ramirez JA. Cardiac diseases complicating community-acquired pneumonia. CurrOpin Infect Dis. 2014 Jun;27(3):295-301

6. Kreutz RP, Bliden KP, Tantry US, Gurbel PA. Viral respiratory tract infections increase platelet reactivity and activation: an explanation for the higher rates of myocardial infarction and stroke during viral illness. $J$ Thromb Haemost. 2005 Sep;3(9): 2108-9.

7. Kreutz RP, Tantry US, Bliden KP, Gurbel PA. Inflammatory changes during the 'common cold' are associated with platelet activation and increased reactivity of platelets to agonists. Blood Coagul Fibrinolysis. 2007 Dec;18(8):713-8.

8. Corrales-Medina VF, Madjid M, Musher DM. Role of acute infection in triggering acute coronary syndromes. Lancet Infect Dis. 2010 Feb;10(2):83-92.

9. Corrales-Medina VC, Musher DM,Shachkina S, Chirinos JA. Acute pneumonia and the cardiovascular system. Lancet. 2013 Feb 9; 381(9865):496-505.

10. BazazR, Marriott HM, Francis
SE, Dockrell DH. Mechanistic links between acute respiratory tract infections and acute coronary syndromes. J Infect. 2013 Jan;66(1):1-17.

11. Miller E, Elkind MSV. Infection and Stroke: an Update on Recent Progress. Curr Neurol Neurosci Rep. 2016 Jan;16(1):2. [PubMed] [Crossref]

12. Collins S.Excess mortality from causes other than influenza and pneumonia during influenza epidemics. Public Health Reports. 1932; 47:2159-2179.

13. Reichert TA, Simonsen L, Sharma A, Pardo SA, Fedson DS, Miller MA. Influenza and the winter increase in mortality in the United States, 1959-1999. Am J Epidemiol. 2004 Sep 1;160(5):492-502.

14. Nunes B, Viboud C, Machado A, Ringholz C, Rebelo-de-Andrade H, Nogueira P, Miller M. Excess Mortality Associated with Influenza Epidemics in Portugal, 1980 to 2004. PLoS One. 2011 Jun 21; 6(6): e20661.

15. Nguyen JL, Yang W, Ito K, Matte TD, Shaman J, Kinney PL. Seasonal Influenza Infections and Cardiovascular Disease Mortality. JAMA Cardiol. 2016 Jun 1;1(3):274-81. [PubMed] [Crossref]

16. Madjid M, Miller CC, Zarubaev VV Marinich IG, Kiselev OI, Lobzin YV, et al. Influenza epidemics and acute respiratory disease activity are associated with a surge in autopsy-confirmed coronary heart disease death: results from 8 years of autopsies in 34,892 subjects. Eur Heart J. 2007 May;28(10):1205-10.

17. Foster ED, Cavanaugh JE, Haynes WG, Yang M, Gerke AK, Tang F, Polgreen PM. Acute myocardial infarctions, strokes and influenza: seasonal and pandemic effects. Epidemiol Infect. 2013 Apr;141(4): 735-44

18. Smeeth L, Thomas SL, Hall AJ, Hubbard R, Farrington P, Vallance P. Risk of myocardial infarction and stroke after acute infection or vaccination. N Engl J Med. 2004 Dec 16; 351(25):2611-8.

19. Clayton TC, Thompson M, Meade TW. Recent respiratory infection and risk of cardiovascular disease: case-control study through a general practice database. Eur Heart J. 2008 Jan;29(1):96-103.

20. Elkind MS, Carty CL, O’Meara ES, Lumley T, Lefkowitz D, Kronmal RA, Longstreth WT Jr. Hospitalization for infection and risk of acute ischemic stroke: the Cardiovascular Health Study. Stroke. 2011 Jul;42(7): 1851-6.

21. Zurrú MC, Alonzo C, Brescacín L, Romano M, Cámera LA, Waisman G, et al. Recent respiratory infection predicts atherothrombotic stroke: case-control study in a Buenos Aires healthcare system. Stroke. 2009 Jun;40(6): 1986-90.

22. Ruane L, Buckley T, Hoo SYS, Hansen PS, McCormack C, Shaw E, et al. Triggering of acute myocardial infarction by respiratory infection. Intern Med J. 2017 May;47(5):522-529. [Crossref]

23. Warren-Gash C, Blackburn R, Whitaker H, McMenamin J, Hayward AC. Laboratory-confirmed respiratory infections as triggers for acute myocardial infarction and stroke: a selfcontrolled case series analysis of national linked datasets from Scotland. Eur Respir J. 2018 Mar 29;51(3): 1701794. [PubMed] [Crossref] 
24. Kwong JC, Schwartz KL, Campitelli MA, Chung H, Crowcroft NS, KarnauchowT, et al. Acute myocardial infarction after laboratoryconfirmed influenza infection. $N$ Engl J Med. 2018 Jan 25;378(4):345-353. [PubMed] [Crossref]

25. Blackburn RM, Zhao $\mathrm{H}$, Pebody R, Hayward AC, Warren-Gash C. Laboratory-confirmed respiratory infections as predictors of hospital admission for myocardial infarction and stroke: time-series analysis of English data for 2004-2015. Clin Infect Dis. 2018 Jan 18; 67(1):8-17. [Crossref]

26. Boehme AK, Luna J, Kulick ER, Kamel H, Elkind MSV. Influenza like illness as a trigger for ischemic stroke. Ann ClinTransl Neurol. 2018 Mar 14; 5(4): 456-63. [Crossref]

27. Fullerton HJ, Hills NK, Elkind MS, Dowling MM, Wintermark M, Glaser CA et al. Infection, vaccination, and childhood arterial ischemic stroke: Results of the VIPS study. Neurology. 2015 Oct 27;85(17): 1459-66. [Crossref]

28. Vejpongsa P, Kitkungvan D, Madjid M, Charitakis K, Anderson HV, Arain S, et al. Outcomes of Acute Myocardial Infarction in Patients with Influenza and Other Viral Respiratory Infections. Am J Med. 2019 Oct; 132(10): 1173-1181. [PubMed] [Crossref]

29. Putot A, Chague F, Manckoundia P, Cottin Y, Zeller M. Post-Infectious Myocardial Infarction: New Insights for Improved Screening. J Clin Med. 2019 Jun; 8(6): 827. doi: 10.3390/jcm8060827

30. Gurfinkel EP, de la Fuente RL, Mendiz O, Mautner B. Flu vaccination in acute coronary syndromes and planned percutaneous coronary interventions [FLUVACS] study. Euro Heart J. 2004 Jan;25(1):25-31.

\section{[PubMed] [ Crossref]}

31. Wu HH, Chang YY, Kuo SC, Chen YT. Influenza vaccination and secondary prevention of cardiovascular disease among Taiwanese eldersA propensity score-matched followup study. PLoS One. 2019 Jul 1; 14(7):e0219172. [Crossref]

32. Udell JA, Zawi R, Bhatt DL, Keshtkar-Jahromi M, Gaughran F, Phrommintikul A et al. Association between influenza vaccination and cardiovascular outcomes in high-risk patients: a meta-analysis. JAMA. 2013 Oct 23;310(16):1711-20.

33. Clar C, Oseni Z, Flowers N, Keshtkar-Jahromi M, Rees K. Influenza vaccines for preventing cardiovascular disease. Cochrane Database Syst Rev. 2015 May 5;(5):CD005050. [PubMed] [Crossref]

34. MacIntyre CR, Heywood AE, Kovoor P, Ridda I, Seale H, Tan T, et al. Ischaemic heart disease, influenza and influenza vaccination: a prospective case control study. Heart. 2013 Dec;99(24):1843-8.

35. Barnes M, Heywood AE, Mahimbo A, Rahman B1, Newall AT, Macintyre CR. Acute myocardial infarction and influenza: a meta-analysis of case-control studies. Heart. $2015 \quad$ Nov;101(21):1738-47. [Crossref]

36. MacIntyre CR, Mahimbo A, Moa AM, Barnes M. Influenza vaccine as a coronary intervention for prevention of myocardial infarction. Heart. 2016 Dec 15;102(24):195356. [Crossef]

37. Grau AJ, Fischer B, Barth C, Ling P, Lichy C, Buggle F. Influenza vaccination is associated with a reduced risk of stroke. Stroke. 2005 Jul; 36 (7):1501-6.
38. Lin HC, Chiu HF, Ho SC, Yang CY. Association of influenza vaccination and reduced risk of stroke hospitalization among the elderly: a population-based case-control study. Int J Environ Res Public Health. 2014 Apr 2; 11(4):3639-49.

39. Lavallee PC, Labreuche J, Fox KM, Lavados P, Mattle H, Steg PG, et al. Influenza vaccination and cardiovascular risk in patients with recent TIA and stroke. Neurology. 2014 May 27;82(21):1905-13.

40. Lee KR, Bae JH, Hwang IC, Kim KK, Suh HS, Ko KD. Effect of Influenza Vaccination on Risk of Stroke: A Systematic Review and Meta-Analysis. Neuroepidemiology. 2017; 48(3-4):103-110. [Crossref]

41. Christiansen CF, Thomsen RW, Schmidt M, Pedersen L, Sørensen HT. Influenza Vaccination and 1-year Risk of Myocardial Infarction, Stroke, Heart Failure, Pneumonia, and Mortality Among Intensive Care Unit Survivors Aged 65 Years or Older: A Nationwide Population-Based Cohort Study. Intensive Care Med. 2019 Jul; 45(7):957-967. [PubMed] [Crossref]

42. Bermudez-Fajardo A, OviedoOrta E. Influenza vaccination promotes stable atherosclerotic plaques in apoE knockout mice. Atherosclerosis. $2011 \mathrm{Jul}$; 217(1):97-105.

43. Veljkovic V, Glisic $S$, Veljkovic N, Bojic T, Dietrich U, Perovic VR, et al. Influenza vaccine as prevention for cardiovascular diseases: possible molecular mechanism. Vaccine. 2014 Nov 12;32(48):656975.

44. Korsun N, Angelova S, Georgieva I. [Influenza.] medinfo.bg. 2016 Jun;14(10):18-22. [in Bulgarian] [nternet]

Please cite this article as: Miteva D. Influenza infection as trigger for acute myocardial infarction and stroke. $J$ of IMAB. 2020 Oct-Dec;26(4):3363-3367. DOI: https://doi.org/10.5272/jimab.2020264.3363

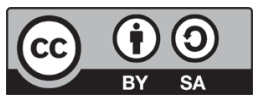

\author{
Address of correspondence: \\ Dr Darina Miteva, PhD \\ Clinic of Pneumology and Phthysiatry, UMHAT "St. Marina” \\ 1, Hristo Smirnenski Blvd., Varna 9010, Bulgaria. \\ E-mail: dari.miteva@abv.bg
}

